\title{
Automated detection of parasitized Cadra cautella eggs by Trichogramma bourarachae using machine vision
}

\author{
Mohammed S. El-Faki ${ }^{*}$, Yuqi Song ${ }^{2}$, Naiqian Zhang ${ }^{2}$, Hamadttu A. El-Shafie ${ }^{1}$, Pan Xin ${ }^{3}$ \\ (1. Date Palm Research Center of Excellence, King Faisal University, Al-Ahsa, Kingdom of Saudi Arabia; \\ 2. Department of Biological and Agricultural Engineering, College of Engineering, Kansas State University, Manhattan, KS 66506, USA; \\ 3. College of Computer and Information Engineering, Inner Mongolia Agricultural University, Hohhot 010018, Inner Mongolia, China)
}

\begin{abstract}
Cadra (Ephestia) cautella (Walker) is a moth that attacks dates from ripening stages while on tree, throughout storage, and until consumption, causing enormous qualitative and quantitative damages, resulting in economic losses. Image-processing algorithms were developed for detecting and differentiating between three Cadra egg categories based on the success of Trichogramma bourarachae (Pintureau and Babaul) parasitization. These categories were parasitized (black and dark red), unparasitized fertile unhatched (yellow), and unparasitized hatched (white) eggs. Color, light intensity, and shape information was used to develop detection algorithms. Two image processing methods were developed based on three randomly selected images and were tested on a larger validation image set of 40 images: (i) segmentation and extractions of color and morphological features followed by Watershed delineation, and is referred to as Algorithm 1 (ALGO1), (ii) finding circular objects by Hough Transformation followed by convolution filtering, and is referred to as Algorithm 2 (ALGO2). ALGO1 and ALGO2 achieved correct classification rates (CCRs) for parasitized eggs of $92 \%$ and $96 \%$, respectively. Their CCRs for unhatched eggs were $48 \%$ and $94 \%$, and for hatched eggs were $42 \%$ and $73 \%$, respectively. Regarding parasitized eggs, both methods performed satisfactorily, but, in general, ALGO2 outperformed ALGO1. These results ensure automatic evaluation of the efficiency of biological control of Cadra cautella by the egg parasitoid Trichogramma bourarachae by quantifying the rate of parasitization. The developed detection methods can be used by producers of biocontrol agents for online monitoring of Trichogramma and similar insect natural enemies during mass production and before release against crop pests. Moreover, with few adjustments these methods can be used in similar applications such as detecting plant diseases.

Keywords: Trichogramma parasitization, Cadra eggs detection, machine vision, date fruit, image processing, objects recognition algorithms, biological control
\end{abstract}

DOI: $10.25165 /$ j.ijabe.20181103.2895

Citation: El-Faki M S, Song Y Q, Zhang N Q, El-Shafie H A, Xin P. Automated detection of parasitized Cadra cautella eggs by Trichogramma bourarachae using machine vision. Int J Agric \& Biol Eng, 2018; 11(3): 94-101.

\section{Introduction}

Date palm (Phoenix dactylifera $\mathrm{L}$.) occupies the top of the fruit crop list in the Kingdom of Saudi Arabia (KSA). The KSA is the world's second largest producer of dates, supplying $17.6 \%$ of the world market ${ }^{[1]}$. Currently, dates production in KSA is about 1 million tons annually. Dates encounter serious economic losses in both quantity and quality caused by warehouse insects. Among the insects attacking stored products is the date moth Cadra cautella. E. cautella (Walker) (Lepidoptera: Pyralidae), which is a common cosmopolitan pest in most of the temperate world as well as in warmer areas. It causes damages to dry fruits, stored grains, and their products ${ }^{[2,3]}$. Cadra cautella is also considered a pest of other stored materials, such as dried fig, wheat flour, chocolate dried fruits, nuts, grain and their processed products ${ }^{[4]}$. Moth larvae can cause considerable damage to stored foods by feeding and contamination with dead bodies and materials they produce

Received date: $2016-10-18 \quad$ Accepted date: $2017-08-20$

Biographies: Yuqi Song, MSc, research interests: image processing, Email: yuqis@ksu.edu; Naiqian Zhang, PhD, Professor, research interest: sensors and control, Email: zhangn@ksu.edu; Hamadttu A. El-Shafie, PhD, research interests: pest management, Email: elshafie@yahoo.com; Pan Xin, PhD, Professor, research interests: artificial intelligence, Email: xinpan@yahoo.com *Corresponding author: Mohammed Salih El-Faki, PhD, Professor, research interests: machine vision. P.O. Box 400, 31982 - Al-Hufof, Al-Ahsa, Kingdom of Saudi Arabia. Tel: +966-135897293, Fax: +966-135897243, Email: elfakim@yahoo.com such as excreta, webbing, silk and feces.

1.1 Biological control using Trichogramma bourarachae

The use of insecticides and other chemical treatments against the pest implies the risk of adverse ecological, toxicological, and economic effects ${ }^{[5]}$. An alternative biological control method to synthetic chemical insecticides for combating E. cautella is to release parasitoid wasps of the genus Trichogramma Westwood into the stored-product environment ${ }^{[6,7]}$. Trichogramma are extremely tiny wasps in the family Trichogrammatidae and they exist naturally in almost every terrestrial habitat and some aquatic habitats as well. These wasps lay their eggs into the E. cautella eggs, thus killing the eggs and preventing their development. Trichogramma species are the most frequently used natural enemies for the control of lepidopteran pests of fruits and cereals in the field ${ }^{[8]}$. Worldwide, egg parasitoids of the genus Trichogramma (Hymenoptera: Trichogrammatidae) have been successfully utilized for bio-control of several Lepidopteran pests. Because of their low host specificity, Trichogramma can be mass reared easily in large numbers and on different natural and factitious hosts ${ }^{[9]}$. More than 150 different species of Trichogramma are known from various biotopes ${ }^{[10]}$. Nine species of Trichogramma are reared in private or government owned insectaries around the world and released annually on an estimated 80 million acres of agricultural crops and forests in 30 countries $^{[8,11]}$.

In Germany and Austria, the control of the Indian meal moth, Plodia interpunctella (Huebner) and the Mediterranean flour moth 
Cadra kuehniella (Zeller) in food processing facilities is achieved by releasing large quantities of Trichogramma evanescens Westwood using the inundative release strategy ${ }^{[12]}$. They parasitize insect eggs, especially eggs of moths and butterflies. Some of the most important caterpillar pests of field crops, forests, and fruit and nut trees are attacked by Trichogramma wasp. In Canada, Trichogramma species are commonly reared and used to control field and glass house insect pests ${ }^{[13]}$. One of the major considerations in the design of an augmentative biological control program using egg-parasitoids is the selection of the parasitoid species or strain, which is in turn determined by its performance in the field ${ }^{[14]}$.

\subsection{Machine vision techniques for evaluating parasitization}

The performance of Trichogramma is assessed by egg parasitism which can be estimated by counting the numbers of parasitized (black and dark red) and unparasitized (white and yellow) eggs in the sample. Currently, this process is done manually through visual counting of parasitized eggs by skilled technicians. Thus, it is subjected to several limitations. Visual detection and counting of eggs is tedious, laborious, time consuming and, therefore, is vulnerable to inaccuracy due to human errors. To overcome these limitations and eliminate errors, this research was carried out to develop procedures for automatic identification and counting of the number of Cadra cautella eggs parasitized by Trichogramma using machine vision technology.

Machine vision is the technology that uses digital image capturing and analysis to automate tasks such as inspection, process control, gauging, counting, and robot guidance in industry, in addition to reading barcodes and optical characters ${ }^{[15-17]}$. While human inspectors can visually inspect parts to judge the quality of workmanship, machine vision systems use advanced hardware and software components to perform these functions at higher speeds and with improved reliably and precision. Image analysis techniques have been applied in the process and food industries for several decades ${ }^{[18]}$ and are being used more frequently in agriculture to discriminate between objects ${ }^{[19-21]}$ and to count items ${ }^{[21,22]}$ with different visual characteristics.

Most automated image systems perform counting by segmenting the item to be counted from the background using various thresholding techniques. Chiu et al. ${ }^{[23]}$ used local adaptive binarization and size filtering to distinguish contour features of bruise areas in Golden Delicious apples for quality control. Back et al. ${ }^{[24]}$ developed an image-based system to monitor the fertilizer application rate of variable-rate applicators, which was estimated from the diameter and number of granules counted. Choi et al. ${ }^{[25]}$ developed a machine vision system for counting the number of citrus fruit drops using an image processing algorithm that included steps for intensity normalization, citrus fruit detection by a logistic classifier, and least square circle fitting. Shariff et al. ${ }^{[22]}$ developed an image analysis algorithm based on fuzzy logic using color, shape and texture features to automatically identify and count the total number of six pest species found in paddy fields. Pearson et al. ${ }^{[21]}$ counted the number of pink bollworm eggs on oviposition pads using histogram features of grayscale images of the pads.

This research aims at automatic evaluation of the efficiency of biological control of Cadra cautella using Trichogramma. The specific objectives were to:

1) Establish a database of digital color images of Cadra eggs after being subjected to Trichogramma parasitization for seven days.

2) Extract features that characterize the parasitized and unparasitized Cadra eggs based on color and shape information in egg images.

3) Design, develop, and test algorithms for segmentation, noise removal, separation of touching eggs, classification, recognition, and counting of parasitized Cadra eggs in digital images.

\section{Materials and methods}

\subsection{Rearing of Cadra cautella}

Cadra cautella was successfully reared in laboratory cultures on standard diet composed of a mixture of a half part of crushed date fruit, one part barley, one and a half parts of broiler feed and one and a half parts layer feed (by weight) as described by $\mathrm{Al}-\mathrm{Azab}^{[26]}$. The sterilized diet was mixed with $400 \mathrm{~mL}$ of glycerol. Newly emerged male and female adults were paired in wooden cage with screen sides. A piece of white paper was put under this cage for collection of eggs. The eggs that fell through the wire mesh were collected in open dishes, then fifteen mg eggs of $E$. cautella were checked under the microscope to remove any impurities or deformed eggs. The E. cautella eggs were transferred into one-liter glass jar containing $200 \mathrm{gm}$ of the previously mentioned sterilized diet. The glass jar was covered with a layer of cheesecloth and placed in an incubator with $50 \%-60 \%$ relative humidity, at $(29 \pm 1)^{\circ} \mathrm{C}$ with 12 light photoperiod.

\subsection{Rearing of Trichogramma bourarachae}

Two species of Trichogramma, namely Trichogramma bourarachae and T. cordubensis (Hymenoptera: Trichogrammatidae), were reared in the lab. The first species is arrhenotokous, producing both females and males through parthenogensis reproduction while the second species produces only females (Thelytokous). These two species are known to be adapted for harsh arid and semi-arid conditions. Two cycles of rearing both Trichogramma and Cadra were carried out so far. Only Trichogramma bourarachae was used in all experiments and the other species was used as backup because these tiny wasps are very sensitive and require special handling during mass production. Colonies of Trichogramma bourarachae used in this study were brought from Egypt. The parasitoids were reared on Cadra cautella (Lepidoptera: Pyralidae) eggs under laboratory conditions. The eggs of Cadra were obtained as described above.

\subsection{Collection of parasitized Cadra eggs used in the experiments}

The method described by Roriz et al. ${ }^{[27]}$ was followed for parasitization of Cadra eggs by $T$. bourarachae with slight modifications. Fifty Cadra eggs were selected from the egg lots, as described above, with the aid of stereoscope (Figure 1) and a fine camel hairbrush. The selected eggs were then carefully glued with agar onto small piece of paper cardboard (sentinel egg cards). The sentinel egg cards were put into small glass tubes $(6 \mathrm{~cm}$ in length and $1 \mathrm{~cm}$ in diameter) and then females of $T$. bourarachae were introduced in these tubes for $24 \mathrm{~h}$ to parasitize the Cadra eggs. At the end of the parasitization period, Trichogramma parasitoids were removed from the glass tubes and the parasitized Cadra eggs on the cards were incubated for $7 \mathrm{~d}$ under the same laboratory conditions for the development of the parasitoids. After the incubation period, the Cadra eggs in each sentinel card could be easily differentiated into three categories; parasitized (black and dark red), unparasitized fertile unhatched (yellow) and unparasitized hatched (white) eggs. The sentinel egg cards each containing the three different categories of Cadra eggs were used for image acquisition.

\subsection{Image acquisition and analysis}

RGB color digital images of Cadra eggs after subjection to 
parasitization by Trichogramma were captured using a DIGI Optika camera mounted on an Optika Tri Zoom Stereoscope with a magnification of $16.5 \mathrm{x}$. The experiment was conducted under laboratory conditions at the Agriculture and Veterinary Training and Research Station, King Faisal University, Al-Ahsa, KSA. All images were acquired under the same imaging conditions (i.e. same background, illumination (1960 lx), resolution $(3200 \times 2400$ pixels $)$, and working distance $(11.5 \mathrm{~cm})$ (Figure 1). The Matlab Mathworks software was used for digital image processing and analysis.

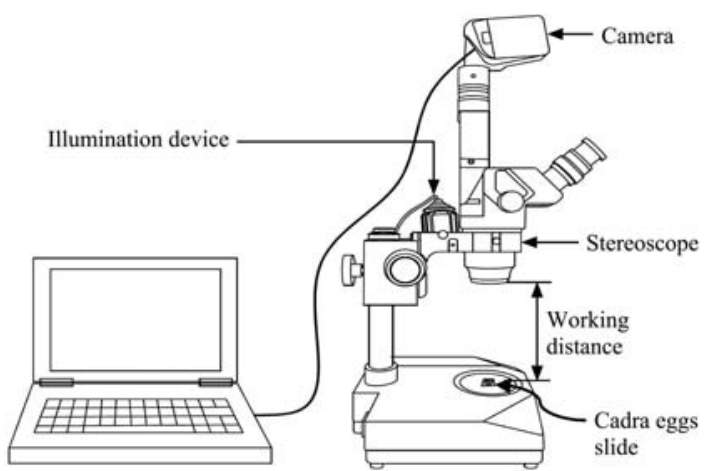

Figure 1 The experimental setup

\section{Development of detection and classification algorithms}

Two image processing methods (algorithms) were used to count cells of different colors in the images. The first method was segmentation and extractions of color and morphological features in the digital images followed by Watershed delineation, and it will be referred to as the Algorithm 1 (ALGO1). The second method used the Hough Transformation function to find circular objects followed by convolution filtering, and it will be referred to as the Algorithm 2 (ALGO2). The algorithms were developed based on three randomly selected images and were then tested on a validation image set of 40 images taken under the same imaging conditions.

\subsection{Algorithm 1}

The overall flowchart of ALGO1 program used for detecting, classifying, and counting different egg categories is shown in Figure 2. The program identified and counted the black and dark red cells (parasitized eggs), the yellow cells (unparasitized unhatched eggs), and the white cells (unparasitized hatched eggs) in an image.

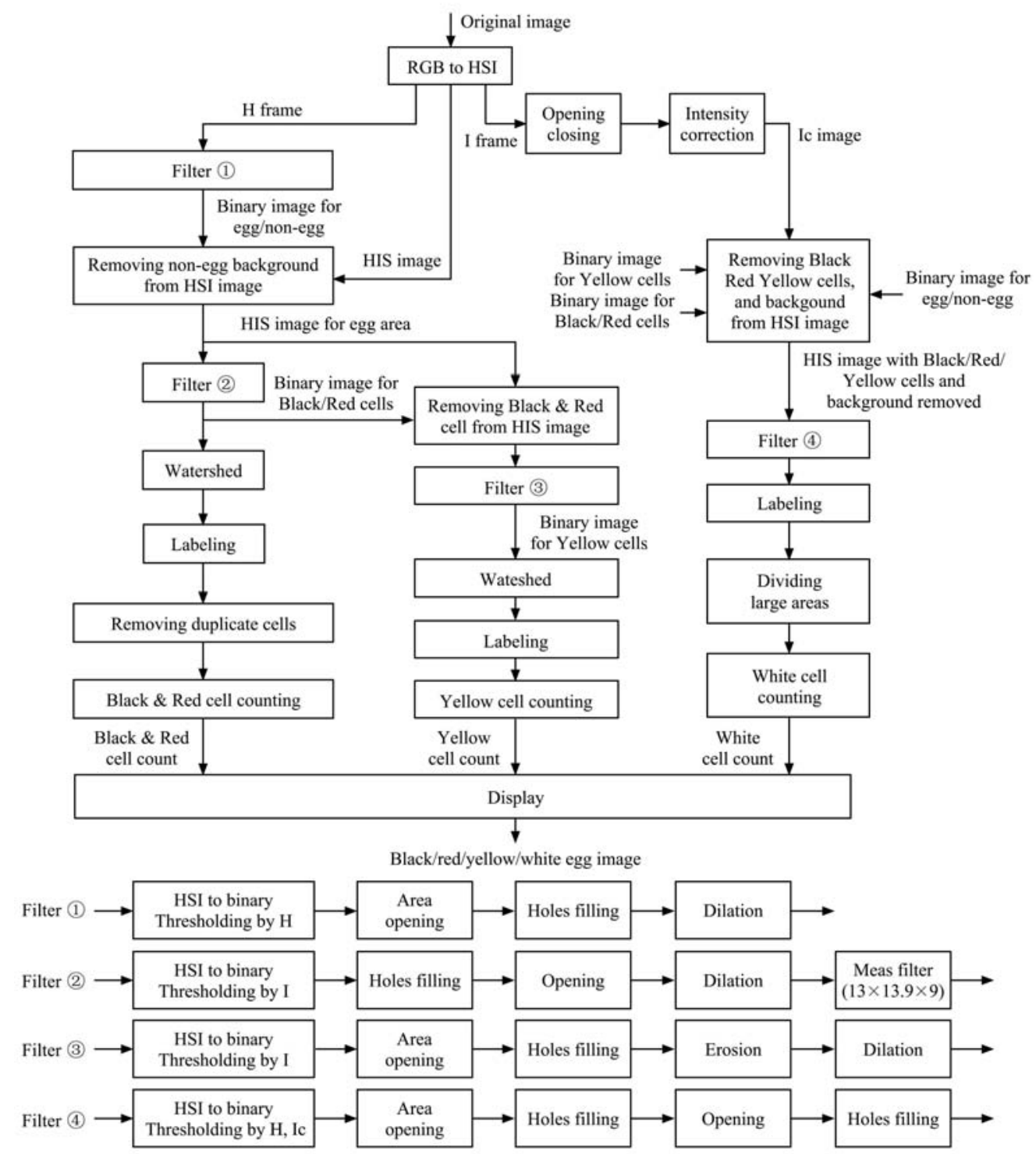

Figure 2 Flowchart of Algorithm 1

3.1.1 Counting black/red (parasitized) eggs

An original RGB color image is shown in Figure 3a. The procedure for identifying and counting the black/red parasitized eggs included the following steps: 
1) Separating black/red cells from the yellow/white cells and background by converting the RGB image to an HSI image, followed by thresholding, morphological filtering, and binarization. The resulting binary image contained only black and red cells (Figure 3b).

2) Identifying individual black/red cells through Watershed delineation and labeling. Distances between the resulting objects were then calculated. Objects with very close distances were then combined. The centers of the resulting objects were identified as the base for counting. Figure $3 \mathrm{c}$ shows the detected parasitized eggs with their centers marked. This included eggs on the edge of the image with only a part visible and eggs touching each other in the image.

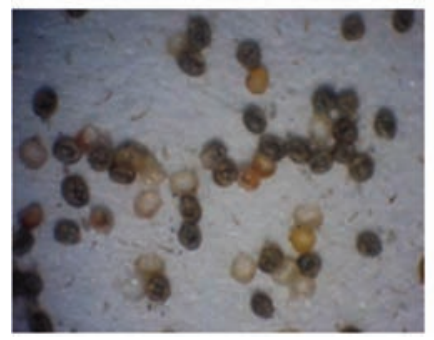

a

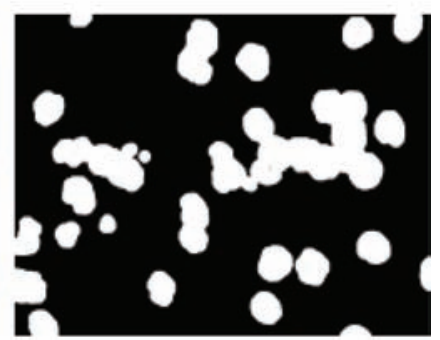

$\mathrm{b}$

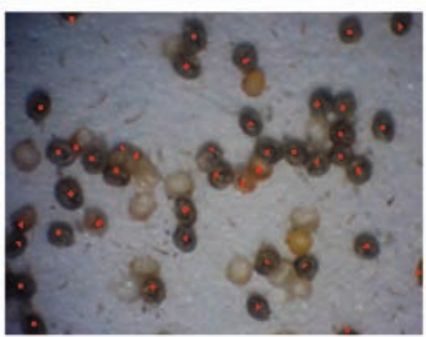

Figure 3 (a) An original RGB color image of Cadra cautella eggs after being subjected to parasitization by Trichogramma bourarachae, (b) the binary image for the black/red (parasitized) eggs after thresholding, morphological filtering, and binarization,

(c) black/red eggs with centers marked through Watershed delineation, labeling, and distance analysis

\subsubsection{Counting yellow eggs (unparasitized unhatched)}

After the black/red eggs were identified, they were removed from the image, leaving only the yellow cells and pixels with similar colors. Thresholding and morphological operations were then conducted to narrow down the possible areas that may contain yellow cells (Figure 4a). Subsequent Watershed analysis and labeling operation were then used to further separate cells. Yellow eggs were finally identified by examining the areas of labeled regions and the distance between potential cell centers (Figure $4 b$ ).

\subsubsection{Counting white eggs}

Among the three categories of eggs, the white eggs (unparasitized hatched eggs) were the most difficult objects to identify. This was mainly due to the uneven illumination within the image. To solve this problem, the intensity of the image was adjusted using a second-order polynomial model based on the average intensities of six $50 \times 50$ pixel sample areas in the image four at the corners and two within the image. The average intensities of the six sample areas were used to calculate the parameters of a complete second-order polynomial surface to be used to adjust the intensities of all pixels in the image. Figure 5a shows an original egg image with non-uniform intensities and the six sample areas in the images selected for intensity adjustment. Figure $5 \mathrm{~b}$ is the intensity-adjusted image. After the adjustment, pixels representing the white eggs were identified through thresholding using the hue and adjusted intensity (Figure 6a). The white eggs were finally identified through filtering, morphological operations, and labeling. Large labeled regions were divided into multiple eggs based on the area of the region. Thus, a labeled region may be considered to contain multiple eggs, as shown by the numbers displayed in Figure 6b. The number of white eggs was then counted.

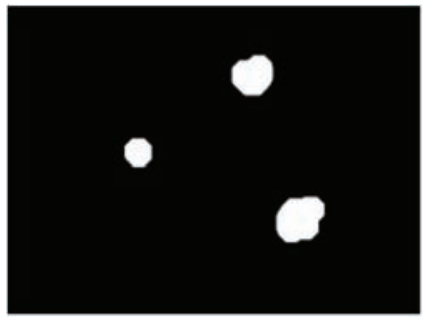

a

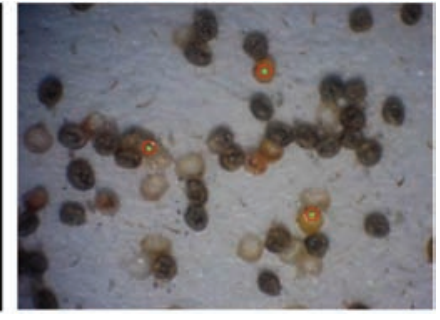

Figure 4 (a) Potential yellow egg areas segmented from the image, (b) yellow eggs extracted through Watershed delineation, labeling, and area/distance analysis

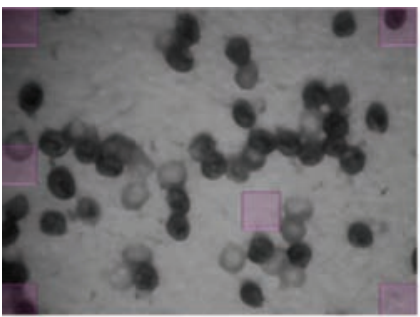

a

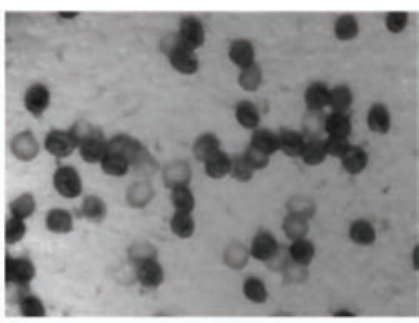

$\mathrm{b}$
Figure 5 Procedure for detecting white eggs: (a) an original image with six sample areas designated to develop the complete second-order polynomial surface for intensity correction,

(b) intensity-corrected image

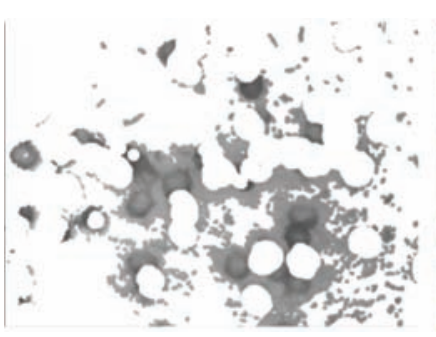

a

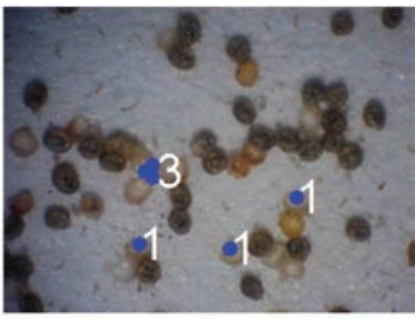

$\mathrm{b}$
Figure 6 (a) Areas of white eggs identified through thresholding, (b) white eggs detected after labeling with blue locations showing the number of multiple eggs

Figure 7 gives the complete classification results using ALGO1 method, showing the original image and an image containing only the detected eggs.

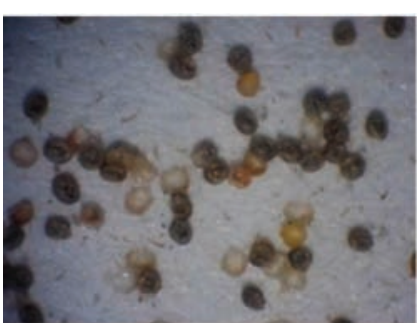

a

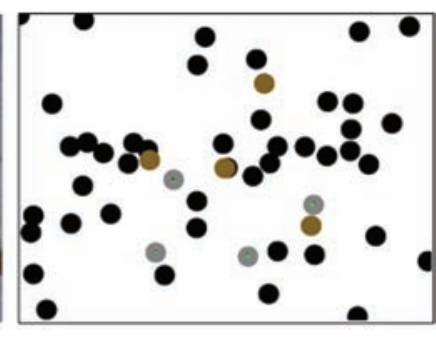

b
Figure 7 Comparison between the original image and the classification result: (a) the original RGB image, (b) the classification result of Algorithm 1 method, showing eggs of all categories- black/red, yellow, and white, in respective colors 


\subsection{Algorithm 2}

The second image processing method emphasized on finding circular objects in the image using the Hough Transformation function. Selection of this function was based on the fact that all the eggs, regardless of their colors, had a circular shape in the images, and the radii of these circles are similar. This method required a minimum amount of image processing. The flow chart of this method is shown in Figure 8.

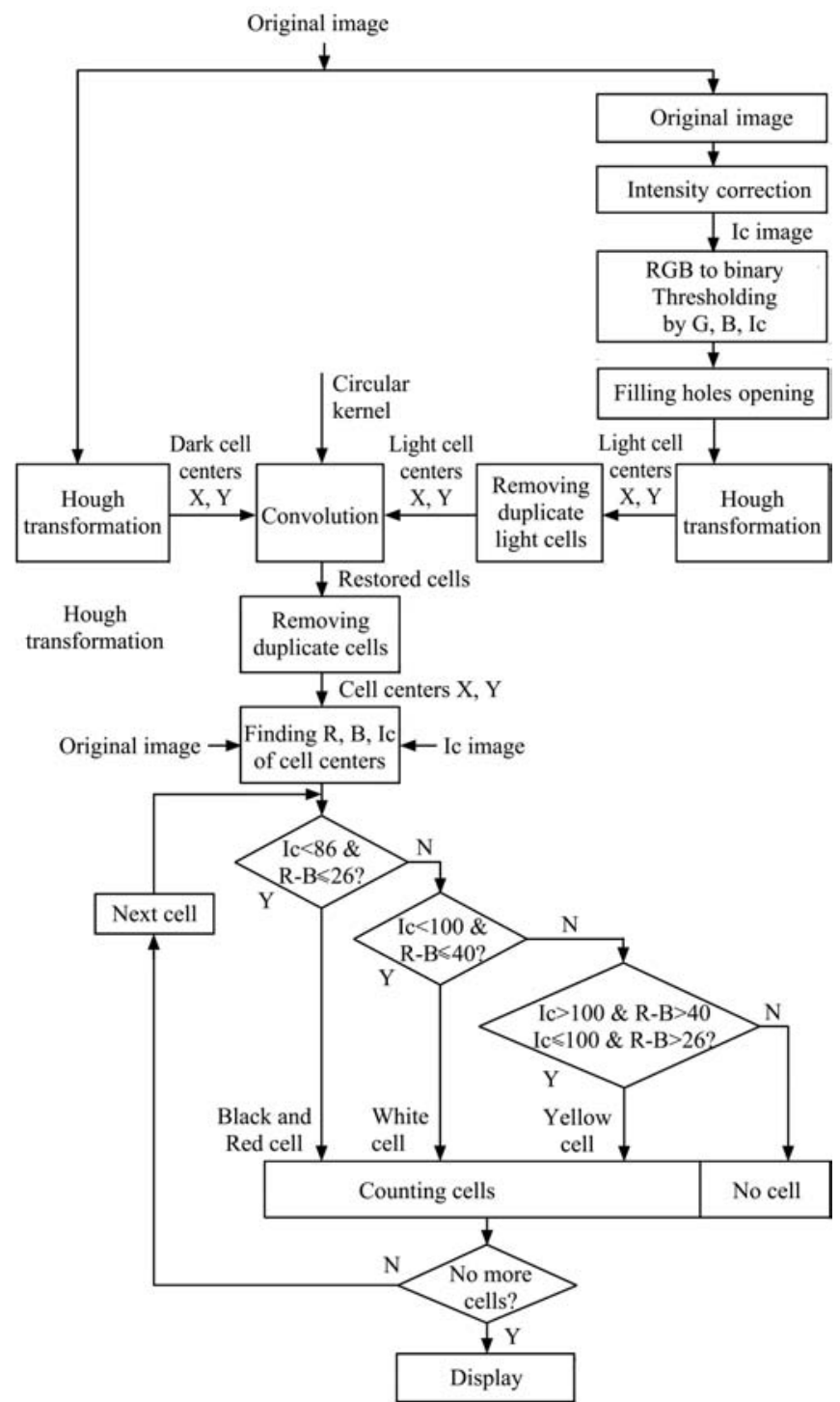

Figure 8 Flowchart of Algorithm 2

For this method, the dark-colored and light-colored cells were first handled separately. Detected cells were then combined, with repeated cells removed. The remaining cells were then classified based on their color and adjusted intensity attributes.

\subsubsection{Finding dark-colored cells}

With properly selected parameters (radius range, sensitivity, and edge threshold), the Hough Transformation function "imfindcircles" in Matlab successfully identified most dark-colored, circular objects in the image. Figure 9 shows the detected dark cells.

\subsubsection{Finding light-colored cells}

ALGO2 had difficulties in finding circular objects with similar color and intensity as the background. Thus, a more extensive preprocessing was performed for the light-colored cells. The preprocessing started with intensity adjustment, which was identical to that used for the ALGO1 method - adjusting the intensity of the entire image using a second-order polynomial surface model as described in Section 2.4.1.3. In order to obtain more smooth adjustment in intensity, the image was first smoothed using an area opening method. Once the intensity was adjusted, the original color image was binarized through thresholding on colors and adjusted intensity. Morphological operations were then performed on the binary image to fill small holes and remove discrete noise pixels. Hough transformation was then performed on the resulting image to identify circular objects. Figure 10a shows the light-colored cells detected through this procedure and Figure $10 \mathrm{~b}$ shows the detected circular objects that most likely were the light colored eggs.

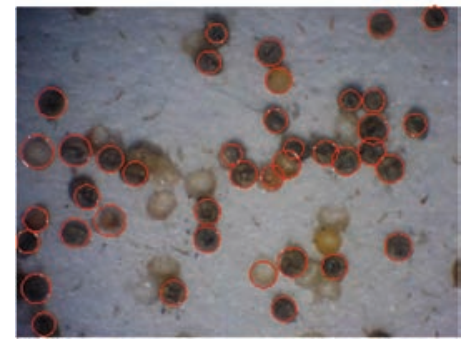

Figure 9 Dark-colored eggs detected using Algorithm 1

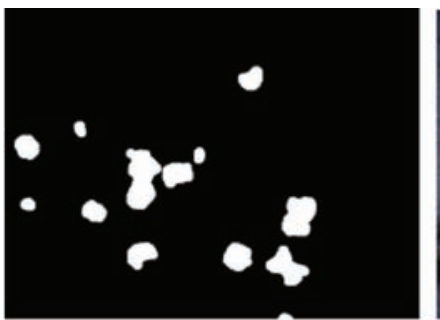

a

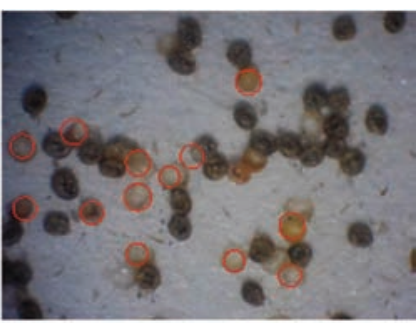

$\mathrm{b}$
Figure 10 (a) Binary image of light-colored eggs detected through intensity correction, thresholding, and morphological filtering,

(b) original color image showing light-colored eggs marked by red circles

\subsubsection{Combining detected cells, classifying, and counting}

After the centers of dark- and light-colored cells were detected, they were merged into a single image. This image was then convoluted with a circular kernel to restore all detected cells. Distances between the cells were then checked. If the distance between two adjacent cells were found to be shorter than the radius of the kernel, one of the cells was deleted. Deleted cells were then removed from the array of detected cells. All remaining cells were then going through a classification procedure based on their adjusted intensity and color attributes. Counts for all categories were updated based on the classification results. To visualize the results, all detected eggs were plotted in their respective colors in an image with white background. Figure 11 compares this image with the original RGB image. It can be seen that, in general, the image processing procedure correctly detected and classified most of the eggs.

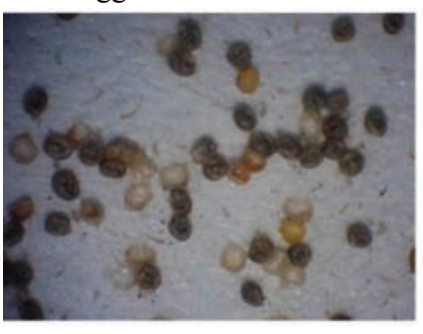

a

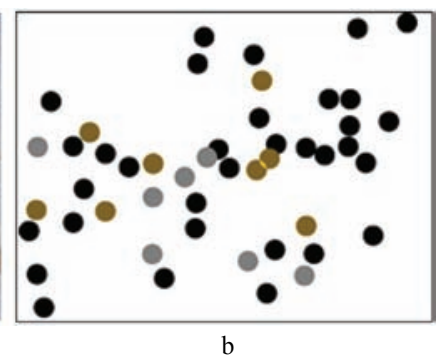

b
Figure 11 (a) Original RGB image, (b) dark-colored (black and dark red), yellow, and white eggs detected by Algorithm 2 method 


\section{Results and discussion}

To evaluate the performance of the algorithms, correct classification rates (CCRs) for individual egg categories and misclassification rates (MCRs) were calculated. Three CCRs are defined as (1) percentage of black/red eggs correctly classified by the program over total number of actual black/red eggs in the image, (2) percentage of yellow eggs correctly classified by the program over total number of actual yellow eggs in the image, and (3) percentage of white eggs correctly classified by the program over total number of actual white eggs in the image. Accompanying these classification rates were misclassification rates across different egg categories. The actual number of eggs in each egg category was calculated manually. Performances of the ALGO1 and ALGO2 methods are evaluated and compared in this section.

\subsection{Performance comparison between ALGO1 and ALGO2}

ALGO1 algorithm was developed using three images, and was tested on the validation image set that included 40 images taken under the same imaging conditions. The total counts of three categories of eggs - black/red, yellow, and white - detected in all 40 images are listed in Table 1. Also included in the table are CCRs for each category, MCRs across categories, numbers of eggs in each category misclassified from background, and numbers of eggs in each category not detected by the algorithm.

ALGO2 was developed using the three images and was tested on the same validation image set as the ALGO1 algorithm. The counts for different categories of eggs and the CCRs and MCRs of various types are presented in Table 2 .

Table 1 Classification and misclassification rates of Cadra (Ephestia) eggs by Algorithm 1 for the validation image set

\begin{tabular}{|c|c|c|c|c|c|c|c|c|c|c|}
\hline \multirow{3}{*}{$\begin{array}{l}\text { Actual eggs } \\
\text { Black }\end{array}$} & \multicolumn{8}{|c|}{ Classified to } & \multirow{2}{*}{\multicolumn{2}{|c|}{ Total }} \\
\hline & \multicolumn{2}{|c|}{ Black } & \multicolumn{2}{|c|}{ Yellow } & \multicolumn{2}{|c|}{ White } & \multicolumn{2}{|c|}{ Background } & & \\
\hline & 1196 & $91.8 \%$ & 10 & $0.8 \%$ & 0 & $0 \%$ & 97 & $7.4 \%$ & 1303 & $100 \%$ \\
\hline Yellow & 111 & $26.3 \%$ & 203 & $48.1 \%$ & 3 & $0.7 \%$ & 105 & $24.9 \%$ & 422 & $100 \%$ \\
\hline White & 5 & $0.9 \%$ & 33 & $6.1 \%$ & 226 & $42.0 \%$ & 274 & $50.9 \%$ & 538 & $100 \%$ \\
\hline Background & 389 & $22.8 \%{ }^{1}$ & 108 & $30.5 \%^{2}$ & 95 & $29.3 \%^{3}$ & \multicolumn{2}{|c|}{ N/A } & 592 & $20.7 \%^{4}$ \\
\hline
\end{tabular}

Note: 1. Percentage of black/red eggs misclassified from background over total number of eggs from all categories detected as black/red.

2. Percentage of yellow eggs misclassified from background over total number of eggs from all categories detected as yellow.

3. Percentage of white eggs misclassified from background over total number of eggs from all categories detected as white.

4. Percentage of all eggs misclassified from background over total number of eggs detected.

Table 2 Classification and misclassification rates of Cadra (Ephestia) eggs by Algorithm 2 for the validation image set

\begin{tabular}{|c|c|c|c|c|c|c|c|c|c|c|}
\hline \multirow{3}{*}{$\begin{array}{l}\text { Actual eggs } \\
\text { Black }\end{array}$} & \multicolumn{8}{|c|}{ Classified to } & \multirow{2}{*}{\multicolumn{2}{|c|}{ Total }} \\
\hline & \multicolumn{2}{|c|}{ Black } & \multicolumn{2}{|c|}{ Yellow } & \multicolumn{2}{|c|}{ White } & \multicolumn{2}{|c|}{ Background } & & \\
\hline & 1251 & $96.0 \%$ & 23 & $1.8 \%$ & 1 & $0.0 \%$ & 28 & $2.1 \%$ & 1303 & $100 \%$ \\
\hline Yellow & 3 & $0.7 \%$ & 395 & $93.6 \%$ & 13 & $3.1 \%$ & 11 & $2.6 \%$ & 422 & $100 \%$ \\
\hline White & 1 & $0.2 \%$ & 15 & $2.8 \%$ & 391 & $72.7 \%$ & 131 & $24.3 \%$ & 538 & $100 \%$ \\
\hline Background & 38 & $2.9 \%{ }^{1}$ & 35 & $7.5 \%^{2}$ & 99 & $19.6 \%^{3}$ & \multicolumn{2}{|c|}{ N/A } & 172 & $7.1 \%^{4}$ \\
\hline
\end{tabular}

Note: 1. Percentage of black/red eggs misclassified from background over total number of eggs from all categories detected as black/red.

2. Percentage of yellow eggs misclassified from background over total number of eggs from all categories detected as yellow.

3. Percentage of white eggs misclassified from background over total number of eggs from all categories detected as white.

4. Percentage of all eggs misclassified from background over total number of eggs detected.

From these tables it can be seen that both ALGO1 and ALGO2 methods were successful in detecting the parasitized Cadra eggs, which is the base for calculating the rate of parasitization. The final results showed that ALGO1 estimated the rate of parasitization to be $53 \%$, while ALGO2 estimated it to be $55 \%$. Comparing these values with the actual rate of parasitization of $57.6 \%$, both methods seemed to have performed well.

\subsection{Performance of ALGO1 and ALGO2 in detecting individual egg categories}

From Tables 1 and 2, it can be seen that both ALGO1 and ALGO2 methods performed satisfactorily in detecting the parasitized Cadra eggs from the validation image set. The CCRs for ALGO1 and ALGO2 were 91.8\% and 96.0\%, respectively. For the detection of yellow unparasitized unhatched Cadra eggs, ALGO2 outperformed ALGO1 with CCRs of $93.6 \%$ and $48.1 \%$, respectively (Tables 1 and 2). The CCRs of white eggs detected by ALGO1 and ALGO2 were $42.0 \%$ and $72.7 \%$ (Tables 1 and 2), respectively. Although ALGO2 performed better than ALGO1 in detecting white eggs, neither method provided completely satisfactory results, probably because that the white Cadra eggs do not possess unique color characteristics, especially when compared with the background. Upon hatching, some unparasitized Cadra eggs became creamy white while others became more transparent and almost colorless, which appeared very similar to the background. Uneven illumination probably worsened the situation even further. The consequence was that some white eggs were misclassified to background and some background areas were misclassified to white eggs.

The ability of the algorithms to detect all eggs in the validation image set without missing eggs was analyzed. This analysis was achieved by comparing the number of missed eggs to the number of eggs counted manually. Missed eggs were eggs that existed in the image but were not detected by the program. In the tables, missed eggs are shown as actual eggs in the image that are classified to background by the program. ALGO1 and ALGO2 methods performed satisfactorily in detecting parasitized Cadra eggs, they missed only $7.4 \%$ and $2.1 \%$ of parasitized eggs, respectively. For the yellow eggs, ALGO2 outperformed ALGO1 by missing only $2.6 \%$ of the actual yellow eggs, while ALGO1 missed $24.9 \%$. The rates for white eggs were $50.9 \%$ and $24.3 \%$ for ALGO1 and ALGO2, respectively. ALGO2 performed better than ALGO1, but neither method provided completely satisfactory 
results. This was the consequence of the creamy or colorless appearance of the white eggs.

The abilities of the algorithms to avoid false detection are also studied. False detection occurred when the program incorrectly recognized a segment of the background as eggs. The false detection rate of $\mathrm{ALGO} 2$ (2.9\%) was much lower than that of ALGO1 (22.8\%) in detecting parasitized Cadra eggs. ALGO2 method also performed better than ALGO1 in detecting yellow eggs. False detection rates of ALGO2 and ALGO1 were 7.5\% and $30.5 \%$, respectively. Although neither algorithms performed well in false detection rate for white cells, ALGO2 still performed better than ALGO1. The false detection rates for white cells by ALGO2 and ALGO1 were $19.6 \%$ and $29.3 \%$, respectively.

Probably the most important measure of the performance of the algorithms is their abilities to classify eggs into correct categories. This was examined by comparing the number of wrongly classified eggs against the number of manually counted eggs. It can be seen that both methods did well for parasitized Cadra (black/red) eggs, but ALGO2 performed better than ALGO1 with wrongly classified black/red eggs into yellow, white, and background categories of $1.8 \%, 0.0 \%$, and $2.1 \%$ for $\mathrm{ALGO} 2$, and $0.8 \%, 0.0 \%$, and $7.4 \%$ for ALGO1. Also ALGO2 performed much better than ALGO1 in classifying yellow eggs. ALGO2 misclassified only $0.71 \%$ of actual yellow eggs to the black/red category, while ALGO1 misclassified 26.3\%. Similarly, ALGO2 misclassified only $2.6 \%$ of actual yellow eggs to the background category, while the rate for ALGO1 was $24.88 \%$.

Generally, ALGO2 method outperformed ALGO1 method in egg detection mainly because ALGO1 depends heavily on color and intensity information whereas the ALGO2 emphasizes on shape (geometric features) information of the objects. When differences in color or intensity were not significant, using shape features may have strengthened the power of detection.

\section{Conclusions}

The study has met the objectives related to establishing a database of color digital images of parasitized Cadra eggs, extracting features characterizing the parasitized and unparasitized Cadra eggs, developing algorithms for segmenting objects from background, removing noise, identifying and separating touching eggs, recognizing, classifying, and counting parasitized and unparasitized Cadra eggs, and determining the rate of parasitization.

Two image processing methods were developed to detect parasitized and unparasitized eggs. Although both methods performed satisfactorily in detecting and counting the number of parasitized eggs and, hence, the rate of parasitization, ALGO2 outperformed ALGO1 in the correct classification rates of all three egg categories. Based on ALGO1 and ALGO2 methods the rates of parasitization were $53 \%$ and $55 \%$, respectively, whereas the actual parasitization rate was $57.6 \%$. The developed detection methods will enable automatic evaluation of biological control of Cadra (Ephestia) cautella using Trichogramma bourarachae. This will facilitate their usage by industries dealing with biological control in online monitoring of Trichogramma and similar insect natural enemies during mass production and before release against crop pests. Although this study aimed at evaluating effectiveness of biological pest control, yet with few adjustments, the developed methods can be used in similar applications, such as detecting plant infestation by insects or their eggs.

\section{Acknowledgements}

We would like to express our thanks to the Date Palm Research Center of Excellence at King Faisal University, Kingdom of Saudi Arabia for funding this research project.

\section{[References]}

[1] Siddiq M, Greiby I. Overview of date fruit production, postharvest handling, processing, and nutrition. In Dates: postharvest science, processing technology and health benefits. 1st edition. Edited by $\mathrm{M}$. Siddiq, S.M. Aleid, and A.A. Kader. John Wiley and Sons, Chichester, United Kingdom, 2014; pp.1-28.

[2] Boshra S A. Effect of gamma irradiation on food consumption, assimilation and digestive enzymes in Cadra cautella (Walker) larvae. Journal of Stored Products Research 2007; 43: 49-52.

[3] Arbogast R T, Chini S R. Abundance of Plodia interpunctella (Hübner) and Cadra cautella (Walker) infesting maize stored on South Carolina farms: Seasonal and non-seasonal variation: J. Stor. Prod. Res, 2005; 41: 528-543.

[4] Singh P, Moore R F. Handbook of Insect Rearing, 1985. Vol. II. 514 p.

[5] Youssef A A, Nasr F N, Stefanos S S, Abou Elkhair S S, Shehata W A, Agamy E, et al. The side effect of plant protection products used in olive cultivation on the hymenopterous egg parasitoid Trichogramma cacoeciae Marchal. J. Appl. Ent., 2004; 128: 593-599.

[6] Schoeller M, Flinn P W. Parasitoids and predators. In: Subramanyam, B., hagstrum, D. W. (Eds.) Alternatives to pesticides in stored-product IPM. Kluwer Academic Publishers, Boston, 2000; pp.229-271.

[7] Steidle J L M, Rees D, Wrigth E J. Assessment of Australian Trichogramma species (Hymenoptera: Trichogrammatidae) as control agents of stored product moths. Journal of Stored Products Research, 2001; 37: 263-275.

[8] Li L Y. Worldwide use of Trichogramma for biological control on different crops: A survey. In Biological Control with Eggs Parasitoids, eds E. Wajnberg and S. A. Hassan, Oxon, U.K., CAB International, 1994; pp.37-53.

[9] Oezder N, Kara G. Comparative biology and life tables of Trichogramma cacoeciae, T. brassicae and T. evanescens (Hymenoptera: Trichogrammatidae) with Cadra kuehniella and Cadra cautella (Lepidoptera: Pyralidae) as hosts at three constant temperatures. Biocontrol Science and Technology, 2010; 20(30): 245-255.

[10] Pino J D. Systematics of the North American species of Trichogramma Westwood (Hymenoptera: Trichogrammatidae). Mem. Entomol. Soc., 1999; 22: 1-287.

[11] Olkowski W, Zhang A. Trichogramma modern day frontier in biological control. The IPM Practitioner, 1990; 12: 1-15.

[12] Schoeller M. Commercial application of parasitoids to control stored-product pests in Germany and Austria. In: Zdarkova E., J. Lukas, J. (Eds.) Cost action 842 Biological control of pests insects and mites, with special reference to Entomophtorales, Proceedings of the first meeting of working group 4: Bio-control of arthropod pests in the stored products, Lisbon 6-7 September 2001; pp.29-32.

[13] Schoeller M, Fields P. Screening of North American species of Trichogramma Westwood (Hymenoptera: Trichogrammatidae) for control of the Indian meal moth, Plodia interpunctella (Huebner) (Lepidoptera: Pyralidae). Proceedings of the eighth International Working Conference of Stored-Product Protection, York, U. K., July 2002.

[14] Hegazi E M, Herz A, Hassan S, Agamy E, Khafagi S, Zaitun A, et al. Naturally occurring Trichogramma species in olive farms in Egypt. Insect Science, 2005; 12: 185-192.

[15] Turek F D. Machine Vision Fundamentals, How to Make Robots See. NASA Tech Briefs, 2011; 35(6): 60-62.

[16] Steger C, Ulrich M, Wiedemann C. Machine vision algorithms and applications. Weinheim: Wiley-VCH, 2008; p.1. ISBN 978-3-52740734-7.

[17] Graves M, Batchelor B G. Machine vision for the inspection of natural products. Springer-Verlag London, 2003; p.5.

[18] Ottavian M, Barolo M, García-Muñoz S. Maintenance of Machine Vision Systems for Product Quality Assessment. Part I. Addressing Changes in Lighting Conditions. Ind. Eng. Chem. Res., 2013; 52(35): 12309-12318.

[19] Pedersen J R. Insects: Identification, damage, and detection. In Storage of Cereal Grains and Their Products, D. B. Sauer, ed. St. Paul, Minn.: AACC, 1992; pp.435-489. 
[20] El-Faki M S. Color discrimination of biological material. Egypt Journal of Applied Science, 2002; 17(7): 451-466 (2002)

[21] Pearson T C, Edwards R H, Mossman A P, Wood D F, Yu P C, Miler E L. Insect egg counting on mass rearing oviposition pads by image analysis. Applied Engineering in Agriculture, 2002; 18(1): 129-135.

[22] Shariff A M, Aik Y, Hong W T, Mansor S, Mispan R. Automated identification and counting of pests in the paddy fields using image analysis. Computers in Agriculture and Natural Resources, 4th World Congress, Proceedings of the International Conference, July 24-26, 2006; pp.759-764. Lake Buena Vista, Florida, USA. ASAE Pub \#701P0606.

[23] Chiu Y C, Chou X L, Grift T E, Chen M T. Automated detection of mechanically induced bruise areas in golden delicious apples using fluorescence imagery. Transactions of the ASABE, 2015; 58(2): $215-225$.
[24] Back S, Yu S, Kim Y, Chung S, Lee K. An image-based application rate measurement system for a granular fertilizer applicator. Transactions of the ASABE, 2014; 57(2): 679-687.

[25] Choi D, Lee W, Ehsani R. Detecting and counting citrus fruit on the ground using machine vision. ASAE paper number 131591603, 2013 Kansas City, Missouri, July 21-24, 2013. (doi: http://dx.doi.org/ 10.13031/aim.20131591603).

[26] Al-Azab A M. Alternative approaches to methyl bromide for controlling Cadra cautella (Walker) (Lepidoptera: Pyralidae). MSc Thesis, College of Agricultural and Food Science, King Faisal University, Saudi Arabia, 2007; $129 \mathrm{p}$.

[27] Roriz V, Oliveira I, Garcia P. Host suitability and preference studies of Trichogramma cordubenesis (Hymenoptera: Trichogrammatidae). Biol Control, 2006; 36: 331-336. 